\title{
Disordered eating attitudes, anxiety levels and self-esteem in female basketball players
}

\author{
M. Michou, V. Costarelli and K. Antonopoulou \\ Department of Home Economics and Ecology, Harokopio University, 70 El. Venizelou Ave, 17671 Kallithea, \\ Athens, Greece
}

Studies have generally suggested a higher frequency of eating disorders among athletes than non-athletes. The role of self-esteem in relation to body image, anxiety levels and disordered eating in athletes of team sports such as basketball, has not yet been thoroughly investigated. The purpose of the current study was to explore the possible differences in disordered eating (DE) attitudes, components of body image, self-esteem and anxiety levels in a group of female basketball players and a group of non-athletes. The inter-relationships of the above parameters have also been examined.

A total of 154 women, 74 Greek basketball players (national and international levels) and 80 women, non-athletes, have been recruited. The mean ages of the two groups were 24.92 (SD 3.8) years and 25.21 (SD 3.4) years for athletes and non-athletes, respectively. All subjects were of normal body weight. The subjects completed the following questionnaires: the Eating Attitudes Test (Eat-26), the Multidimentional Body-Self Questionnaire (MBRSQ), the State-Trait Anxiety Inventory (STAI) and the Rosenberg's Self-Esteem Scale (RSE).

The results indicate that $11 \%$ of the basketball players demonstrated DE attitudes; however, there were no significant differences in DE attitudes prevalence between the two groups. The analysis of specific components of the body image questionnaire (MBRSQ) has revealed that athletes scored significantly higher in fitness orientation $(P=0.021)$ and fitness evaluation $(P=0.019)$, which was anticipated. The State-Trait Anxiety Inventory (STAI) and the Rosenberg's Self-Esteem Scale (RSE) have shown no significant differences between athletes and non-athletes. Finally, the analysis has also revealed that in the group of 154 young women (athletes and non-athletes), the women with DE attitudes had higher levels of anxiety and scored significantly more in components of the body image questionnaire (MBRSQ) such as appearance orientation $(P=0.002)$, health evaluation $(P=0.026)$, health orientation $(P=0.000)$ and illness orientation $(P=0.003)$.

Due to the reported susceptibility of athletes to developing eating disorders, the investigation of the possible role of anxiety levels, selfesteem and disturbed body image as well their inter-relationships in the aetiology of disordered eating, warrants further investigation. 